\title{
De l'enseignement catholique au foulard musulman: la laïcité scolaire entre débats et mutations en France, 1984-2004*
}

\section{ISMAIL FERHAT}

Profesor de la Université de Picardie Jules Verne (Francia). Correo electrónico: Ismail.ferhat@u-picardie.fr. El autor es magíster y doctor en Sciences-po París (Francia). Entre sus últimas publicaciones tenemos: "Les gauches et les réformes éducatives: Quel(s) regard(s) des sciences sociales sur un couple complexe?” en Carrefours de l'éducation No. 41 (2016) y "Une valeur qui (dés) unit? Le PS et la laïcité, du Congrès d'Épinay à la Commission Stasi” en Parlements No. 23 (2016).

Recibido: 26 de agosto de 2015

Aprobado: 10 de junio de 2016

Modificado: 23 de junio de 2016

Artículo de investigación científica

DOI: http://dx.doi.org/10.15648/hc.30.2016.9

Este artículo forma parte del proyecto: "Axe 1 du Laboratoire CAREF" financiación propia. 
De l'enseignement catholique au foulard musulman: la laïcité scolaire entre débats et mutations en France, 1984-2004

\title{
Résumé
}

La laïcité est un aspect historique du système éducatif en France. Cependant, ce pays a récemment connu sur ce sujet des mutations aussi rapides que majeures. En 1984, celui-ci était encore lié à l'affrontement séculaire entre une partie des républicains et les écoles catholiques. En 2004, il était désormais cristallisé par la question de l'interdiction du foulard islamique dans les établissements scolaires publics. L'article vise à présenter les débats intellectuels et idéologiques qui ont accompagné cette transformation.

Mots-clés: laïcité, France, islam, école, catholicisme, république.

De la enseñanza católica al velo musulmán: la laicidad escolar entre debates y mutaciones en Francia, 1984-2004

\section{Resumen}

la laicidad es un aspecto histórico del sistema educativo francés. Sin embargo, recientemente este país ha conocido al respecto mutaciones tan rápidas como importantes. En 1984, el tema todavía se vinculaba al enfrentamiento secular entre una parte de los republicanos y las escuelas católicas. A partir de 2004, cristalizaría en el asunto de la prohibición del velo islámico en las instituciones escolares públicas. Este artículo tiene como objetivo presentar los debates intelectuales e ideológicos que han acompañado esta transformación.

Palabras clave: laicidad, Francia, islam, escuela, catolicismo, república.

From Catholic schools to muslim veil: debates and mutations of secularism in French education system, 1984-2004

\begin{abstract}
Laïcité" (or militant secularism) is a historical part of the French education system. This country has nevertheless experienced some recent and rapid changes on this issue. In 1984, it was still linked to the traditional conflict between a part of Republicans and Catholic schools. In 2004, it was focused on banning the Muslim veil in public schools. The article aims at presenting the intellectual and ideological controversies which have been fuelled by this mutation.
\end{abstract}

Key words: secularism, France, islam, catholicism, school, republic. 


\section{Do ensino católico ao velo muçulmano: a laicidad escolar entre debates e muta- ciones no França, 1984-2004}

\section{Resumo}

A laicidad é um aspecto histórico do sistema educativo francês. Porém, recentemente este país tem conhecido ao respeito mutaciones tão rápidas como importantes. Em 1984, o tema ainda se vinculava ao confronto secular entre uma parte dos republicanos e as escolas católicas. A partir de 2004, cristalizaria no assunto da proibição do velo islâmico nas instituições escoares públicas. Este artigo tem como objetivo apresentar os debates intelectuais e ideológicos que têm acompanhado esta transformação.

Palavras-chave: laicidad, França, islão, escola, catolicismo, república.

\section{INTRODUCTION}

Dans son usage courant en France, la notion de laïcité renvoie à une triple dimension. La première, de type juridique, renvoie à une séparation des pouvoirs publics et des institutions religieuses, définie par la loi du 9 décembre 1905 et devenue principe constitutionnel le 27 octobre 1946. Cette approche est notamment privilégiée par la haute cour de justice administrative française, le Conseil d'Etat, dans un rapport publié en 2003 intitulé «un siècle de laïcité». Le deuxième aspect de la laïcité a une acception philosophique, celle de la liberté de conscience et du refus par conséquence du contrôle de l'espace public par le religieux. L'article $1^{\text {er }}$ de la constitution du 4 octobre 1958 -celle actuellement en vigueur- stipule ainsi que la République «respecte toutes les croyances». Troisième aspect, la laïcité renvoie à une sensibilité politique et militante. Dans l'histoire contemporaine française, il n'est pas rare de rencontrer des organisations qui se revendiquent ou se nomment «laïques». C'est ce que l'on peut synthétiser sous la notion de «galaxie laïque» ${ }^{1}$. Celle-ci regroupe des organisations politiques (républicains, radicaux, socialistes, communistes) philosophiques (franc-maçonnerie laïque, libre-pensée, rationalistes) syn-

1 Ismail Ferhat, "Le Parti socialiste et la galaxie laïque", Le Parti socialiste d'Epinay au Panthéon, 1971-1981, eds. Noëlline Castagnez y Gilles Morin (Rennes: Presses Universitaires de Rennes, 2015), 189-202. 
dicales (syndicalisme enseignant notamment) et associatives (Ligue de l'enseignement, éducation populaire). Historiquement, la laïcité -dans ses trois dimensions- a été liée à un autre sujet identitaire du modèle politique français, l'école. En effet, les deux relèvent de «l'Etat tuteur», qui a pour volonté d'éduquer et de transformer le corps social ${ }^{2}$. Le système éducatif a constitué depuis le XIXe siècle un des sujets centraux de l'application du principe de laïcité.

Au début des années 1980, près d'un siècle après les grandes lois scolaires de 1881-1882, la laïcité scolaire paraissait être un sujet à la fois réglé et archaïque. Le mouvement de Mai 68 avait déstabilisé aussi bien la galaxie laïque, le système éducatif public que l'Eglise catholique. Ce sentiment d'une laïcité scolaire comme d'un sujet dépassé était formulé par un historien reconnu, Alfred Grosser, dans une tribune parue début 1977. Celle-ci, selon lui, comportait des «affirmations dogmatiques et simplificatrices» ${ }^{3}$. Pourtant, en une vingtaine d'années, le débat sur la laïcité à l'école a connu une profonde -et inattendue- mutation en France. Le 12 juillet 1984, le président de la République François Mitterrand a retiré le projet de loi Savary. Celui-ci a constitué le dernier affrontement majeur entre un pouvoir de gauche et l'enseignement privé catholique en France. Deux décennies plus tard, le 15 mars 2004, après un débat parlementaire et public particulièrement virulent, une loi interdisait les signes religieux ostensibles pour les élèves des établissements scolaires publics. Celle-ci était liée à la question du foulard islamique porté par certaines élèves de collèges et de lycées.

Cette mutation a entraîné des débats politiques et intellectuels nombreux sur le contenu même de la notion de laïcité. Celle-ci a entraîné une production scientifique extrêmement importante. Le catalogue de la bibliothèque de Sciences-po Paris (une des plus riches en sciences sociales en France) permet de mesurer cette montée. En 1983, la notion de «laïcité» apparaît dans deux titres de publications en français. En 2004, elle se retrouve dans vingt-un titres. L'intérêt médiatique a aussi été fortement accru. La base de

2 Pierre Rosanvallon, L'Etat en France de 1789 à nos jours (Paris: Le Seuil, 1990).

3 Alfred Grosser. "L'avenir de la liberté". Le Monde, Paris, 11 janvier, 1977. 
données des archives de la télévision publique française (INA) montre une telle évolution, avec deux mentions de «laïcité» dans les archives audiovisuelles conservées en 1982, contre quatorze pour l'année 2004. Par-delà cet aspect quantitatif, qui est déjà en soi révélateur de l'importance du sujet dans la société française, il convient de s'interroger sur les mutations concrètes de la laïcité à l'école en France de 1984 à 2004. Quelles significations et enjeux celle-ci a-t-elle recouverts durant ces deux décennies? Quelles évolutions a-t-elle connues entre ces deux dates?

La réflexion se structure en trois moments intellectuels successifs qui ont existé dans cette période de vingt ans. Tout d'abord, il sera examiné la fin des affrontements traditionnels autour de la laïcité scolaire durant les années 1980 (I). Ceux-ci sont remplacés par la question nouvelle de l’islam à l'école, cristallisée par «l'affaire du foulard» en 1989 (II). Celle-ci aboutit à une fragmentation du monde intellectuel en deux camps, particulièrement vive de la fin des années 1990 à la loi de 2004 (III).

\section{Du CATHOLICISME À L'ISLAM: UNE RÉORIENTATION DE LA LAÏCITÉ À L'ÉCOLE (1984-1989)}

Le retrait du projet de loi Savary, le 12 juillet 1984, vient clore plusieurs cycles importants de l'histoire politique et scolaire de la France. Ceux-ci, au nombre de trois, peuvent être classés selon leur ampleur historique. Le premier cycle, le plus court, est celui de ce projet Savary lui-même. En 1981, le programme à l'élection présidentielle de François Mitterrand prévoyait un dispositif nouveau de gestion des écoles privées bénéficiant d'un financement public (le GSPULEN, ou Grand service public unifié de l'Education nationale). Dès le 4 août 1982, le premier projet gouvernemental sur le sujet avait abandonné l'idée d'une intégration à l'Etat de ce secteur éducatif privé. Le Ministre de l'Education nationale Alain Savary a en effet privilégié la conciliation entre enseignement public et enseignement privé ${ }^{4}$ Or, cette position rejetée par les représentants de ces deux institutions ${ }^{5}$. Le retrait de ce projet en 1984 entraîne la fin de la promesse

5 Serge Hurtig (eds.), Alain Savary: politique et honneur (Paris: Presses de Sciences-po, 2002). 
présidentielle, le pouvoir de gauche ne rouvrant plus le sujet de l'étatisation des écoles catholiques.

Le second cycle est plus long: il s'est ouvert en 1959, avec le vote de la loi Debré. Celle-ci permettait un financement des écoles privées (le plus souvent catholiques) par l'Etat. En échange, celles-ci s'engageaient à respecter les programmes d'enseignement publics et à laisser au libre choix des élèves et des parents les cours religieux. La loi avait été acceptée non sans tensions au sein de la majorité parlementaire de droite en 1959. En 1960, une gigantesque pétition avait été organisée, recueillant près de 12 millions de signatures contre la loi Debré. Les forces de gauche, regroupées dans le cadre d'un groupe de pression laïque, le CNAL (Comité national d'action laïque), ont à plusieurs reprises affirmé leur volonté de revenir sur ce texte législatif. Ce fut notamment le cas lors d'un colloque le 13 et 14 mai 1972. Or, un quart de siècle après le vote de la loi Debré, les sondages montraient que l'opinion avait largement accepté ce nouveau cadre- y compris dans l'électorat des partis de gauche. Le gouvernement socialiste lui-même avait commandé des enquêtes d'opinion confirmant ce changement de perception $^{6}$. A partir de 1984, plus aucune force politique d'importance (qu'elle soit de droite ou de gauche) ne s'oppose à la loi Debré et à l'enseignement privé financé par l'Etat'. Jean-Pierre Chevènement, Ministre socialiste de l'Education nationale (1984-1986) affirme ainsi sa volonté d'appliquer loyalement cette loi ${ }^{8}$. Après qu'une majorité de droite gagne les élections législatives en mars 1986, le nouveau Ministre de l'Education nationale, René Monory, déclare dès le 20 mars à la radio Europe 1 qu’il ne souhaite pas rouvrir le dossier de l'école privée?.

Le troisième cycle est probablement le plus fondamental pour comprendre les mutations des années ultérieures. En effet, 1984 clôt un affrontement séculaire entre l'Eglise catholique et les forces politiques de gauche sur la question de l'école. Ces tensions avaient en effet commencé avec les

6 “Compte rendu d'un sondage d'opinion sur la question de l'enseignement privé", Ministère de l'Education nationale, 25 février, 1982, 3 SV 5, Fondation nationale des sciences politiques. Bruno Poucet, La liberté sous contrat. Une histoire de l'enseignement privé (Paris: Fabert, 2009).

8 Bernard Toulemonde, Petite histoire d'un grand ministère (Paris: Albin Michel, 1988), 259-260.
9 Le Monde, "Monory ne veut pas rouvrir le dossier de l'école privée", Le Monde, Paris, 22 mars, 1986. 
réformes scolaires de la IIIe République (rétablissement du monopole d'Etat sur les diplômes, scolarisation obligatoire et gratuite dans le premier degré, laïcisation des personnels et des locaux scolaires). Une partie des catholiques accusaient le système éducatif républicain d'être "sans Dieu» les programmes scolaires ayant progressivement été expurgés des références à la morale religieuse ${ }^{10}$. En sens inverse, le sentiment anticlérical était virulent chez une partie des républicains, qui s'opposaient frontalement à l'Eglise catholique ${ }^{11}$. Les affrontements autour du projet de loi Savary, comme le rappelait l'historien Emile Poulat, a été un exemple tardif de ce conflit traditionnel ${ }^{12}$.

La fermeture de ces trois cycles en 1984 réoriente la question de la laïcité sur de nouveaux enjeux. C'est en particulier la question de l'islam qui réoriente le questionnement de la société française -et des pouvoirs publics- dans leurs rapports au religieux. Cette évolution est liée à plusieurs facteurs, qui n'ont là aussi rien d'exclusifs. Le premier est démographique. Jusqu'au tout début des années 1980, les populations musulmanes présentes en France étaient d'abord composées d'étrangers, le plus souvent membres du monde ouvrier. C'était par l'étiquette de «travailleurs immigrés» que les musulmans sont perçus, notamment dans la presse ${ }^{13}$. Lors de cette décennie, l'apparition d'une génération de musulmans nés en France affaiblit cette perception en termes de classe sociale. En juillet 1983, deux journaux importants, le quotidien Le Monde et l'hebdomadaire l'Express, consacrent des enquêtes fouillées aux musulmans français ${ }^{14}$. Les sciences sociales elles-mêmes reflètent ce tournant. En 1987 apparaît une première étude savante sur les musulmans de France, menée par un islamologue de renom, Gilles Kepel ${ }^{15}$. Une seconde raison réside dans la crainte croissante de l'intégrisme musulman, symbolisé par la révolution islamique en Iran de 1979. Ceci se traduit notamment, dans une partie de l'opinion et des

10 Pierre Ognier, Une école sans Dieu? 1880-1895. L'invention d’une morale läque sous la IIIe République (Toulouse: Presses universitaires du Mirail, 2008).

11 Jacqueline Lalouette, La République anticléricale, XIXe-XXe siècles (Paris: Seuil, 2002).

12 Emile Poulat, "Liberté scolaire, laïcité républicaine", La Pensée No 237 (1984), 42-52.

13 Alexandre Bousseageon, "L'impossible ramadan", Témoignage chrétien, París, 20 aout, 1979.

14 Emmanuel Flandre y Jean-Claude Pattacini, "Paris: l'islam des pauvres", L'express, Paris, 15 juillet, 1983; Jean-Marie Durand-Souffland, "Musulmans en France”, Le Monde, Paris, 13 juillet, 1983.

15 Gilles Kepel, Les Banlieues de l'islam. Naissance d'une religion en France (Paris: Seuil, 1987). 
médias, par la crainte d'une montée de l'islam en France. Cette peur est notamment symbolisée dans la presse par la figure récurrente du symbole de la République, Marianne, affublé d'un foulard islamique ${ }^{16}$. Dès 1983, une grève comprenant de nombreux travailleurs de culture musulmane, dans l'industrie automobile à Poissy (région parisienne), a suscité des accusations d'intégrisme ${ }^{17}$. Dans les partis politiques, cette crainte devient explicite à partir du milieu de la décennie, comme le montre la déclaration d'une cadre du Parti socialiste, Françoise Gaspard, le 12 mars 1987 au sein d'une réunion de son parti:

«La montée des intégrismes, en France et dans le monde, est un péril pour les libertés et je crois que les Français le sentent à travers notamment -il ne faut pas le nier dans bien des quartiers où il y a des maghrébins- la montée de l'intégrisme musulman».

Chez certaines organisations laïques, une telle crainte est aussi présente: ainsi, le 9 septembre 1985, la conférence de presse du Grand Orient de France dénonce l'intégrisme islamique avec virulence.

Cette réorientation des controverses religieuses de la société française se renforce durant les années 1980. Une note interne du Ministère de l'Education nationale, en 1985, montre notamment que la question des élèves issus de l'immigration devient un enjeu important des politiques éducatives ${ }^{18}$. Plus globalement, le Ministère de Jean-Pierre Chevènement de 1984 à 1986 voit la montée des interrogations sur la manière dont le système éducatif français intègre (ou pas) les élèves immigrés, la diversité culturelle, les mondes extra-européens. Ainsi, le ministre commande au spécialiste de l'islam Jacques Berque un rapport novateur sur la place de l’immigration dans l'école, qui est établi en 1985.

16 Alec Hargreaves, "Marianne musulmane: de l'exclusion (post)coloniale à l'intégration républicaine", La République en représentations: autour de l'œuvre de Maurice Agulhon, eds. in Maurice Agulhon, Annette Becker, Evelyne Cohen (Paris: Publications de la Sorbonne, 2006), 59-68.

25217 Claude Chetucci, Nicolas Hatzfeld, "L'administration du Travail et les conflits collectifs: Citroën et Talbot (1982-1983)", Travail et Emploi No.111 (2007), 31-38.

18 "Les migrants et les élèves en difficulté", Note de la direction générale des enseignements scolaires, 30 janvier, 1986 (19880108/5, Archives nationales). 
Dans ce contexte en mutation, plusieurs organisations et intellectuels souhaitent redéfinir le contenu de la laïcité en général, et à l'école en particulier. C'est notamment le cas des organisations laïques, qui après 1984 multiplient les journées de réflexion. Peuvent être cités le colloque du CNAL du 23-24 novembre 1985 et le colloque du Grand Orient de France (GODF, principale obédience maçonnique française) le 18 et 19 janvier 1986. Les deux évènements proposent de réorienter la laïcité vers de nouveaux enjeux (médias, communication, économie) qui avaient été jusque-là négligés par la galaxie laïque. La grande association d'éducation populaire française, la Ligue de l'enseignement, va plus loin encore dans la réflexion sur le contenu de la laïcité. A son congrès de Lille du 1er-3 avril 1986, intitulé «laïcité 2000», elle affirme un refus du «repli de l'idée laïque sur l'école». Elle y condamne de même «la cristallisation sur le monde catholique comme adversaire idéal et éternel». La charge est violente et achève de montrer les divisions au sein même de la galaxie laïque divisions qui préexistaient certes aux années 1980 mais qui s'expriment plus fortement durant cette décennie. Un ouvrage d'entretiens dirigé par le responsable laïcité de Ligue de l'enseignement, Guy Gauthier, confirme cette volonté de rénover sa doctrine laïque ${ }^{19}$. Le livre est de plus original: il donne la parole à des intellectuels se réclamant de différentes confessions (notamment catholique et musulmane), ce qui était jusque-là rare dans la galaxie laïque. L'idée d'une approche de la laïcité qui dépasserait le seul cadre scolaire semble cependant intéresser une partie de cette sensibilité. Ainsi, le Comité national d'action laïque, dans son colloque du 12 mars 1988 à Créteil, reprend cette idée d'une réflexion qui ne se centrerait plus sur l'école ${ }^{20}$.

Une partie du monde religieux aboutit à une même conclusion, celle d'une reformulation de la laïcité qui prendrait en compte les évolutions de la société française. C'est notamment le cas de la Fédération protestante de l'enseignement, une organisation regroupant les écoles privées protestantes. Cette structure publie, juste après le retrait du projet Savary, une tribune appelant à: «Une lä̈ité ouverte, respectueuse de toutes les convictions et de toutes les

19 Guy Gauthier, La laïcité en miroir (Paris: Edilig, 1985).

20 Philippe Bernard, "Le CNAL ne veut plus réduire la laïcité à la seule question scolaire", Le Monde, 15 mars, 1988 
cultures, qu'il s'agisse de la culture ouvrière, que l'école ignore, ou des cultures issues de l'immigration mais aussi des racines judéo-chrétiennes de la culture majoritaire qu'il ne faut plus occultens ${ }^{21}$. Une telle idée se retrouve dans l'entretien qu'aborde le sociologue Jean Baubérot au journal protestant La réforme $e^{22}$. La notion de «laïcité ouverte» constitue une expression qui a un rôle important dans les débats sur la laïcité par la suite. Elle se popularise alors même que la réorientation sur l'islam se confirme en 1989 du fait de «'affaire de Creil».

\section{L'affaire de Creil (1989): rupture ou Cristallisation D'une NOUVELLE «LAÏCITÉ»?}

Le colloque du 22 avril 1989 sur la laïcité organisé par le journal catholique la Croix et auquel participent la FEN (Fédération de l'Education nationale, principale organisation syndicale enseignante en France de 1946 à 1992) et la Ligue aurait pu être un symbole d'apaisement de la société française sur ce sujet. En effet, les organisations qui se sont opposées lors du conflit sur l'école privée entre 1981 et 1984 étaient présentes à cet évènement. Il s'agissait d'une volonté revendiquée de dialogue, qui semble confirmer les transformations qui ont eu cours durant cette décennie. Les années 19881989 sont cependant aussi le moment d'évènements semblant confirmer la remontée de l'intégrisme religieux. Le premier fait, interne à la France, est l'incendie par un catholique extrémiste d'un cinéma parisien le 22 octobre 1988. Celui-ci était accusé de projeter le film de Martin Scorcèse, «La dernière tentation du Christ». Le deuxième, de nature internationale, est la fatwa de religieux chiites iraniens, le 14 février 1989, contre l'écrivain Salman Rushdie. Le 26 février 1989, un millier de personnes défilent à Paris à l'appel de la Voix de l'islam (une association musulmane extrémiste) contre cet intellectuel.

Dans ce contexte déjà tendu par la crainte de l'intégrisme religieux, intervient une affaire qui a eu un rôle fondamental dans les mutations des rapports entre laïcité et école en France. Le 18 septembre 1989, trois collégiennes musulmanes viennent en portant un foulard à leur établissement

21 Anne-Marie Goguel, “Un jugement de Salomon”, Réforme, 4 août, 1984.

22 Interview de Jean Baubérot, Réforme, 7 juin, 1986. 
Gabriel-Havez, à Creil (département de l'Oise). La législation en vigueur à l'époque n'interdisait pas formellement de tels signes religieux dans l'espace scolaire. Cependant, le dirigeant du collège décide, alors que la communauté éducative est très divisée par cette affaire, de ne pas accepter les collégiennes en cours. Cette affaire, à l'origine purement locale, devient nationale: le 4 octobre 1989, une dépêche de l'agence de presse publique (l'AFP), révèle en effet celle-ci à l'opinion. Un véritable emballement médiatique a lieu, avec une couverture par la presse, la radio et la télévision particulièrement intense d'octobre à décembre 198923. Le Ministre de l'Education nationale Lionel Jospin (1988-1992) refuse de trancher en faveur de l'exclusion. Il sollicite le 4 novembre l'avis de la haute cour de justice administrative, le Conseil d'Etat. Celui-ci rend un avis le 27 novembre 1989, qui prône une gestion établissement par établissement des signes religieux ostensibles des élèves. Cette position est reprise par la circulaire ministérielle du 12 décembre 1989. L'avis du Conseil d'Etat est fondamental: en effet, il souhaite établir un équilibre entre deux positions apparemment irréconciliables sur la laïcité scolaire. D’une part, il rappelle que

«le port par les élèves de signes par lesquels ils entendent manifester leur appartenance à une religion n'est pas par lui-même incompatible avec le principe de laïcité». D'autre part, il note que «cette liberté ne saurait permettre aux élèves d'arborer des signes d'appartenance religieuse qui (...) constitueraient un acte de pression, de provocation, de prosélytisme ou de propagande».

Cette tentative d'équilibre ne suscite pas l'apaisement, bien au contraire: elle ouvre un débat politique, juridique et intellectuel sur la laïcité scolaire, d'autant plus virulent que ses conditions sont nouvelles.

Ainsi que le note un document interne au service communication du Ministère de l'Education nationale, les positions du monde politique et intellectuel français ne correspondent pas aux habituelles lignes de clivage. En effet, les camps politiques, syndicaux et associatifs sont profondément

23 "Note de synthèse du Service d'information et de diffusion", 6 novembre, 1989 (104 APO 9, Office universitaire de recherche socialiste). 
divisés par l'affaire de Creil. Deux sensibilités sont repérées par le document: une première serait une «conception classique » de la laïcité, une seconde serait une «conception renouvelée, tolérante» de celle-ci ${ }^{24}$. L'affaire de Creil cristallise les positionnements intellectuels des années 1980 sur le sujet. Une tribune d'intellectuels prestigieux (Élisabeth Badinter, Régis Debray, Alain Finkielkraut, Élisabeth de Fontenay et Catherine Kintzler) parait dans l'hebdomadaire Nouvel Observateur du 2 novembre 1989. Elle assimile l'acceptation du foulard islamique dans l'école publique à «Munich»c'est-à-dire la capitulation diplomatique des démocraties face au $3^{\text {ème }}$ Reich en 1938 en Tchécoslovaquie. Le 6 novembre 1989, des militants laïques, des intellectuels et des féministes (notamment Gisèle Halimi, grande figure de la lutte pour le droit à l'avortement) se réunissent dans un meeting à Paris pour demander l'exclusion des élèves voilées des écoles publiques. Cet évènement symbolise un tournant: la question du droit des femmes est désormais liée de manière de plus en plus systématique à l'évocation de la laïcité. Une position aussi dure se retrouve chez un des spécialistes universitaires de l'islam les plus réputés de l'époque, Bruno Etienne, dans un ouvrage paru en $1989^{25}$. Selon lui, «la laïcité est contournée par des tactiques minoritaires s'affirmant avec force aujourd'buì.

Face à cette affirmation d'une fermeté laïque, une autre sensibilité dénonce l'exclusion des élèves et le risque d'une laïcité qui conduirait à stigmatiser l'islam. C'est notamment le cas des militants et d'universitaires du PSU (Parti socialiste unifié) qui dans un dossier consacré à l'affaire de Creil. Ils y dénoncent une laïcité devenue une «coquille vide», voire un moyen détourné de critiquer une population musulmane le plus souvent présente dans les classes populaires ${ }^{26}$. Une telle position se retrouve dans une partie de l'extrême-gauche, qui assimile l'exclusion des foulards islamiques du domaine scolaire à une forme de racisme et d'intolérance ${ }^{27}$. C'est dans ce contexte que certains intellectuels relancent une réflexion critique sur la laïcité -et ses mutations- qui avait été initiée dans les années 1980. C’est en

24 "Note de synthèse du Service d'information et de diffusion", 6 novembre, 1989 (104 APO 9, Office universitaire de recherche socialiste).

25 Bruno Etienne, La France et l'islam, (Paris: Hachette, 1989).

26 "Quand la laïcité se voile la face...", Rouge et vert, 18 novembre, 1989.

27 Béatrice Rayman, Albert Rochal, "Intégration ou assimilation?”, Critique communiste nº1 (1989). 
particulier le cas de Jean Baubérot, qui publie en 1990 un ouvrage intitulé Pour un nouveau pacte lä̈que. Il y défend la nécessité d'une adaptation du modèle laïc français à une situation de pluralité culturelle et religieuse croissante (immigration, déclin du catholicisme, montée de nouvelles religiosités). Une telle réflexion se retrouve chez les sociologues de la religion, ainsi Danièle Hervieu-Léger, qui souligne la crise des institutions et pratiques religieuses traditionnelles ${ }^{28}$. Or, celles-ci, paradoxalement, avaient simplifié le régime de laïcité, qui supposait des cultes suffisamment installés pour réguler la sphère religieuse.

Cette réflexion, qui ne se concentre pas encore la question spécifique de l'école, se radicalise du fait du contexte international. A partir de 1992, l'Algérie, ancienne colonie française et pays d'origine d'une importante diaspora en France, connaît une sanglante guerre civile entre régime militaire et islamistes de l'AIS (Armée islamique du Salut) puis du GIA (Groupes islamiques armés). Ce conflit, d'une grande brutalité (enlèvements, tortures, viols, massacres de villages entiers), entraîne l'exode de nombreux algériens -notamment des intellectuels francophones- vers la France. Le conflit déborde au sens propre en France, avec une vague d'attentats en 1995 à Paris, revendiqués par les GIA. Plus grave, des jeunes Français de culture musulmane sont impliqués dans des actes terroristes: c'est le cas de Khaled Kelkal, terroriste tué par les forces de l'ordre le 29 novembre 1995 près de Lyon. Dès lors, le conflit algérien et la montée de l'islamisme radical en France même suscitent aussi forte division de la part de l'intelligentsia française. Une grande partie d'entre elle dénonce l'islamisme, perçu désormais à la fois comme une menace internationale et comme un risque intérieur. C'est notamment le cas d'intellectuels comme Bernard-Henri Lévy, qui publie un reportage sur son voyage en Algérie dans le quotidien Le Monde le 8 et 9 janvier 1998. D’autres dénoncent une vision négative et discriminante des musulmans en Europe. C'est notamment le cas de l'historien Benjamin Stora et du philosophe Mohamed Harbi. Ceux-ci soulignent que:

28 Danièle Hevieu-Léger, La religion en mouvement: le pèlerin et le converti, (Paris: Flammarion, 1999). 
«La condamnation légitime de la violence et de l'intégrisme ne peut justifier tous les amalgames. Rien n'est plus dangereux, dans une Europe recroquevillée sur elle-même, que de voir un intégriste dans chaque musulman et un islamiste dans chaque immigré» $^{29}$.

Ces débats sur l'articulation entre société française, islam et laïcité sont peu affectés par l'éphémère retour de la question de l'école privée catholique en 1993-1994. La majorité parlementaire de droite, élue le 28 mars 1993, s'était en effet engagée dans une augmentation des moyens alloués aux établissements privés. Une manifestation des militants laïques, le 16 janvier 1994, entraîne l'abandon de ce projet. 10 ans après l'affaire Savary, cet ultime affrontement entre galaxie laïque et enseignement catholique confirme la mutation des débats sur la laïcité scolaire.

\section{1997-2004: LA CONFIRMATION DE DEUX SENSIBILITÉS EN MATIÈRE DE LAÏCITÉ SCOLAIRE}

Le début des années 1990 avait vu un certain gel des débats entrainés par l'affaire de Creil de 1989. Les raisons en sont à la fois juridiques et politiques. Le 2 novembre 1992, un premier contentieux opposant la famille d'une élève voilée à un établissement scolaire est tranché par le Conseil d'Etat. Il s'agit d'une collégienne exclue d'un établissement de Montfermeil (commune au nord de Paris) en vertu du règlement intérieur, qui interdisait les signes religieux ostensibles chez les élèves. Le Conseil d'Etat annule la décision d'exclusion. Cependant, cette décision ne suscite pas les passions, comme a pu le faire la première affaire de foulard islamique en établissement scolaire en 1989. En effet, les ministres de l'Education nationale Jack Lang (socialiste, 1992-1993) et François Bayrou (centre-droit, 1993-1997), ne souhaitent pas relancer un débat politiquement coûteux et socialement vif. Certes, François Bayrou avait édicté une circulaire, le 20 septembre 1994, qui refusait «d'accepter à l'école la présence de signes si ostentatoire que leur signification est précisément de séparer certains élèves des règles de vie commune de l'école». Cependant, elle stipulait aussi que «l'adhésion à la règle est

29 Rouge et vert, 1 février, 1995. 
souvent le résultat d'un travail de persuasion». La circulaire Bayrou ne différait pas radicalement de l'avis du Conseil d'Etat et de la circulaire Jospin de 1989: il s'agissait d'éviter un nouvel abcès politico-médiatique sur la laïcité à l'école.

Cette volonté d'une solution souple et progressive était de plus liée à une approche plus globale des politiques publiques en matière religieuse. Le Ministère de l'Education nationale n'était qu'une des pièces de l'action gouvernementale envers l'islam. Le ministère de l'intérieur (chargé depuis la loi de 1905 des relations avec les cultes) avait en effet multiplié les tentatives dans les années 1990 de créer un interlocuteur institutionnel, capable de représenter les musulmans de France ${ }^{30}$. Près de trois tentatives de la part de ce Ministère ont lieu durant la décennie de créer un tel organisme. Peuvent être cités le Conseil de réflexion sur l'islam en France en mars 1990, le Conseil représentatif des musulmans de France en septembre 1993, la consultation sur l'islam de France d'octobre 1999.

Un tel intérêt public nourrit la réflexion sur la laïcité en général, et son application à l'école en particulier. Les deux sensibilités intellectuelles qui s'étaient structurées durant l'affaire de Creil précisent leurs positions respectives. Le sociologue Jean Baubérot publie ainsi en 1997 La morale lä̈que contre l'ordre moral. Cet ouvrage s'intéresse à la manière dont le système éducatif public a créé, des lois Ferry à 1918, une morale laïque. Celle-ci, selon lui, était profondément libérale et visait à sortir d'un ordre moral traditionnel. Cette idée se retrouve dans une partie du monde politique et associatif. Ainsi, la ministre de la culture (1997-2000), Catherine Trautmann, appelle à une défense d'une «laïcité ouverte» capable de libéralisme, lors du congrès du PS le 22 novembre 1997. De même ligue de l'enseignement créé en en février 1997, une Commission «Islam et Laïcité» (initialement Laïcité et Islam). Celle-ci permet une rencontre inédite entre intellectuels de gauche et penseurs de culture musulmane. Un exemple de production liée à cette commission est l'ouvrage dialogué entre Alain Gresh (journa-

30 Jocelyne Cesari, Être musulman en France: Associations, militants et mosquées (Paris: Karthala/ IREMAM, 1994), 137-158; Bernard Godard, La question musulmane en France. Un état des lieux sans concessions (Paris, Fayard, 2015). 
liste du Monde diplomatique) et l'islamologue suisse Tariq Ramadan, L'islam en questions, publié en 2000. Cette sensibilité refuse toute interdiction pour les élèves de porter des signes religieux ostensibles, à commencer par le voile.

Une autre sensibilité intellectuelle sur la laïcité scolaire refuse une telle approche. Elle se structure notamment autour d'universitaires et auteurs comme les philosophes Catherine Kintzler et Henri Pena-Ruiz, l'essayiste Régis Debray, les historiens Claude Nicolet et Maurice Agulhon, les journalistes Eric Conan ou Patrick Kessel. Elle se retrouve dans une série d'associations laïques, comme l'UFAL (union des familles laïques, née en 1988). En 1998 est publié l'ouvrage de Catherine Kintzler, Tolérance et lä̈cité. Elle y affirme la primauté de la séparation entre structure étatique et religion, condition nécessaire de la coexistence des croyances et noncroyances. Cette idée se retrouve dans l'ouvrage d'Henri Pena-Ruiz, Dieu et Marianne. Philosophie de la laïcité, publiée en 1999. Celui-ci note que les religions ont eu tendance à vouloir régenter la société et l'Etat: la laïcité doit dès lors être capable d'imposer à celles-ci des règles communes à l'ensemble du corps social. Cette approche refuse en conséquence toute notion de «laïcité ouverte», qui laisserait entendre a contrario que la laïcité ne serait pas ouverte en soi. Cette sensibilité ne se limite pas à la question des signes religieux ou des règlements intérieurs dans les établissements scolaires. Elle encourage aussi à l'enseignement laïque des faits religieux dans le système éducatif. C'est notamment le sens du rapport de Régis Debray au Ministère de l'Education nationale de 2002, qui souhaite une approche sécularisée des religions dans le milieu scolaire ${ }^{31}$.

Ces deux sensibilités ne tardent pas à s'affronter. Dans un premier temps, ce n'est pas l'islam qui a servi de sujet d'affrontement, mais un autre aspect de la laïcité scolaire, à savoir les langues régionales et minoritaires, qui a suscité le conflit. En 1999, la France signe la Charte européenne des langues régionales ou minoritaires, un texte de l'Union européenne visant à protéger ces pratiques linguistiques au sein des Etats membres. Le texte

31 Régis Debray, "L`enseignement du fait religieux dans lécole laïque” (Paris, Ministère de l'Education nationale, 2002). 
suscite un débat passionné en France, le Conseil constitutionnel bloquant en 1999 son adoption après avoir été consulté par la Présidence de la République. En effet, la Constitution précise que le français est la langue officielle unique de la France. En 2001, le Conseil d'Etat a cassé le projet d'intégration dans le service public des écoles Diwan, des établissements scolaires totalement en langue bretonne. Les partisans d'une application ferme du principe de laïcité se sont montrés globalement hostiles à une reconnaissance accrue des langues minoritaires dans l'école publique. Ils organisent d'ailleurs, le 13 mai 2000, un colloque à la Sorbonne contre la Charte européenne. Y interviennent des intellectuels comme Henri Péna-Ruiz ou Pierre-André Taguieff (spécialiste de philosophie politique). En sens contraire, les partisans d'une «laïcité ouverte» se montrent plus ouverts à l'intégration accrue des langues minoritaires au nom notamment du multiculturalisme. Ce lien entre défense d'une société pluriculturelle et laïcité ouverte est explicité par l'historienne Esther Benbassa, spécialiste des minorités de la société française ${ }^{32}$. De nombreux universitaires en sciences sociales rejoignent cette seconde position: peuvent être cités Jean Baubérot, Béatrice Mabilon-Bonfils, Geneviève Zoïa. Les affrontements entre les deux sensibilités sont, dès le début des années 2000, sont particulièrement marqués.

A partir de 2001, le débat entre ces deux sensibilités intellectuelles sur la laïcité scolaire revient -et désormais presque exclusivement- à l'islam. Le contexte explique largement ce réalignement. Les attentats du 11 septembre aux Etats-Unis mettent en lumière le phénomène d'un terrorisme islamiste de masse. En France même, le sujet de la laïcité est relancé à cette période, avec une nouveauté: une partie des mouvements politiques de droite s'en réclament désormais. Ce tournant politique est symbolisé par un rapport du député (de droite) François Baroin, «Pour une nouvelle laïcité», rendu public en mai 2003. Fait important, les divisions sur la question de la laïcité scolaire ne recoupent pas les frontières des partis politiques. Ainsi, le Parti socialiste, passé dans l'opposition en 2002, se révèle profondément clivé par le sujet ${ }^{33}$. De plus, la question de la place

32 Esther Benbassa, La République face à ses minorités (Paris: Fayard, 2004).

33 Ismail Ferhat, "Une valeur qui (dés)unit? Le PS et la laïcité, du Congrès d'Épinay à la Commission 
de l'islam au sein du système scolaire est relancée par une nouvelle affaire de voile islamique, dans un lycée de la banlieue nord de Paris. Deux sœurs sont en effet exclues en octobre 2003 pour port d'un foulard. La couverture médiatique est particulièrement intense les élèves étant les filles d'un avocat, Laurent Lévy, qui a exercé des responsabilités dans le mouvement antiraciste MRAP.

Les affrontements entre les deux conceptions de la laïcité scolaire deviennent particulièrement tranchés durant les années 2003-2004. Du 3 juillet au 11 décembre 2003, une commission appelée par le Président de la République Jacques Chirac et dirigée par l'ancien ministre Bernard Stasi, étudie la question de l'interdiction éventuelle des signes religieux ostensibles dans les écoles publiques. Une loi est votée en ce sens, et est promulguée le 15 mars 2004. Les universitaires et intellectuels qui avaient alimenté les débats sur la laïcité depuis les années 1980 étaient parfois parties prenantes du processus politique. Ainsi, Jean Baubérot était membre de la commission Stasi il y était résolument hostile à ce qui allait devenir la loi du 15 mars 2004. Certains chercheurs en sciences sociales assimilent la commission Stasi et la demande d'interdiction des signes religieux ostensibles dans l'enseignement public à une forme de «prohibition», voire de racisme

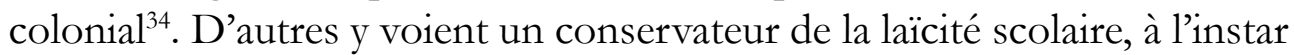
de la sociologue Françoise Lorcerie ${ }^{35}$. Il est à noter qu'une partie non-négligeable de la galaxie laïque était soit réservée, soit même hostile à une loi interdisant les signes religieux ostensibles à l'école. C'était notamment le cas de la Ligue de l'enseignement, par la voix de son secrétaire adjoint, Pierre Tournemire ${ }^{36}$. Le 16 décembre 2013, une majorité des syndicats enseignants, la grande fédération laïque de parents d'élèves (la FCPE), la Ligue de l'enseignement établissent un communiqué commun contre une éventuelle loi en ce sens.

Stasi”, Parlements No 23 (2016), 193-205.

34 Simona Tersigni, "«Prendre le foulard»: les logiques antagoniques de la revendication", Mouvements No 30 (2003) 116-122; Charlotte Nordmann (eds.), Le foulard islamique en questions (Paris, Éditions Amsterdam, 2004).

35 Françoise Lorcerie, “La 'loi sur le voile’: une entreprise politique”, Droit et société No 68 (2008), 53-74.

36 Pierre Tournemire, "Une loi qui serait inopportune”, Idées en mouvement No 110, (2003). 
La question de ce qui apparait être un durcissement religieux à l'école publique suscite cependant aussi la crainte d'une partie du monde intellectuel. Elle est décrite dans deux ouvrages de nature différente. Un premier est écrit par des enseignants intervenant dans des établissements à forte population pauvre et musulmane. Il alerte sur la montée de l'antisémitisme, du sexisme et de l'intégrisme chez certains élèves se réclamant de l'islam, faisant de ces écoles des «territoires perdus de la République» ${ }^{37}$. Le second texte est un rapport officiel, dirigé par un haut fonctionnaire du Ministère de l'Education nationale, Jean-Pierre Obin ${ }^{38}$. Il décrit une évolution similaire, avec la montée d'une conception identitaire et affichée de l'appartenance religieuse en milieu scolaire. Le rapport suscite une publication collective, signée par certains intellectuels et universitaires laïques, comme l'écrivain Thierry Jonquet ou la politiste Jeanine Costa-Lascoux ${ }^{39}$. Les deux camps qui s'étaient formés durant les années 1980 étaient désormais en affrontement ouvert. En 2003-2004, il y a un durcissement des clivages intellectuels, politiques et militants sur le sujet, désormais entièrement tourné vers l'islam. Ainsi, les questions de la «burka» (voile intégral), des menus de cantines scolaires ou des mères voilées ont suscité des débats houleux sur la période allant de 2004 à aujourd'hui. De fait, la sensibilité critique vis-à-vis de la laïcité accuse l'interdiction des signes religieux ostensibles dans le système scolaire public d'être raciste ${ }^{40}$. La loi du 15 mars 2004 marque de ce point de vue à la fois un pic de tension et l'ouverture d'une nouvelle étape de débats sur la laïcité scolaire.

\section{ConClusion}

Les années 1984-2004 ont été un moment tournant pour les interactions entre laïcité, école et religions en France. En effet, elles auraient pu être le lieu d'un apaisement de rapports qui ont été historiquement passion-

37 Emmanuel Brenner, Les Territoires perdus de la république. Antisémitisme, racisme et sexisme en milieu scolaire (Paris: Mille et une nuits, 2002).

38 Jean-Pierre Obin, Les signes et manifestations d'appartenance religieuse dans les établissements scolaires (Paris, Ministère de l'Education nationale, 2014).

39 Collectif, L'école face à l'obscurantisme religieux (Paris: Max Milo, 2006).

40 Béatrice Mabilon-Bonfils, Geneviève Zoïa, La laïcité au risque de l>Autre (La Tour d>Aigues: Éditions de l>Aube, 2014). 
nelles dans la société française. La fin des débats sur l'existence d'écoles privées subventionnées par l'Etat a été en effet rapide et pérenne jusqu'à aujourd'hui. Cependant, ce sujet a été remplacé par celui de l'islam comme moteur des conflits conceptuels, philosophiques et politiques autour de la laïcité scolaire. Si certains évènements (affaire du foulard islamique en 1989, commission Stasi en 2003) ont été des moments de cristallisation, la nouveauté intellectuelle de ces deux décennies réside dans un autre aspect. Il existe désormais en France deux sensibilités nettement identifiées. Une prône une application toujours plus ferme du principe de laïcité scolaire- y compris chez les élèves ou chez les parents d'élèves. Une autre s'en tient à une perception plus souple, voire à un compromis avec les religions. Le fossé entre ces deux conceptions a fini par créer deux camps opposés, qui s'affrontent régulièrement. Ultime paradoxe, la laïcité scolaire, qui est au cœur du modèle républicain français, en est aussi devenu un des aspects les plus polémiques.

\section{Bibliografía}

\section{Libro y artículo en libro:}

Baubérot, Jean. Les 7 laïcités françaises. Paris: Maison des Sciences de l'Homme, 2015.

Benbassa, Esther. La République face à ses minorités. Paris: Fayard, 2004.

Brenner, Emmanuel. Les Territoires perdus de la république. Antisémitisme, racisme et sexisme en milieu scolaire. Paris: Mille et une nuits, 2002.

Collectif. L'école face à l'obscurantisme religieux. Paris: Max Milo, 2006.

Cesari, Jocelyne. Être musulman en France: Associations, militants et mosquées. Paris: Karthala/IREMAM, 1994.

Ferhat, Ismail. "Le Parti socialiste et la galaxie laïque", Le Parti socialiste d'Epinay au Panthéon, 1971-1981, editado por Noëlline Castagnez, Gilles Morin. Rennes: Presses Universitaires de Rennes, 2015, 189-202.

Ferhat, Ismail. "Une valeur qui (dés)unit? Le PS et la laïcité, du Congrès d'Épinay à la Commission Stasi”, Parlements No 23 (2016), 193-205.

Debray, Régis. L'enseignement du fait religieux dans l'école lä̈que Paris, Ministère de l'Education nationale, 2002.

Etienne, Bruno. La France et l'islam. Paris: Hachette, 1989.

Gauthier, Guy. La laïcité en miroir. Paris: Edilig, 1985.

264 Mabilon-Bonfils, Béatrice. Geneviève Zoïa, La laïcité au risque de lıAutre (La Tour d`Aigues: Éditions de l>Aube, 2014) 
Godard, Bernard. La question musulmane en France. Un état des lieux sans concessions. Paris: Fayard, 2015.

Hargreaves, Alec. "Marianne musulmane: de l'exclusion (post)coloniale à l'intégration républicaine", La République en représentations: autour de l'œuvre de Maurice Agulhon, editato por Maurice Agulhon, Annette Becker, Evelyne Cohen. Paris: Publications de la Sorbonne, 2006, 59-68.

Hevieu-Léger, Danièle. La religion en mouvement: le pèlerin et le converti. Paris: Flammarion, 1999.

Hurtig, Serge (eds.). Alain Savary: politique et honneur. Paris: Presses de Sciences-po, 2002.

Kepel, Gilles. Les Banlieues de l'islam. Naissance d'une religion en France. Paris: Seuil, 1987.

Lalouette, Jacqueline. La République anticléricale, XIXe-XXe siècles. Paris: Seuil, 2002.

Lorcerie, Françoise. La Politisation du voile. L'affaire en France, en Europe et dans le monde arabe. Paris: L'Harmattan, coll. Confluences Méditerranée, 2004.

Nordmann, Charlotte (eds.). Le foulard islamique en questions. Paris: Éditions Amsterdam, 2004.

Obin, Jean-Pierre. Les signes et manifestations d'appartenance religieuse dans les établissements scolaires. Paris: Ministère de l'Education nationale, 2004.

Ognier, Pierre. Une école sans Dieu? 1880-1895. L'invention d'une morale laïque sous la IIIe République. Toulouse: Presses universitaires du Mirail, 2008.

Poucet, Bruno. La liberté sous contrat. Une histoire de l'enseignement privé. Paris: Fabert, 2009.

Rosanvallon, Pierre. L'Etat en France de 1789 à nos jours. Paris: Le Seuil, 1990.

Savary, Alain. En toute liberté. Paris: Hachette, 1985.

Toulemonde, Bernard. Petite histoire d'un grand ministère. Paris: Albin Michel, 1988.

\section{Artículo}

"Monory ne veut pas rouvrir le dossier de l'école privée". Le Monde, Paris, 22 mars, 1986.

Bernard, Philippe. "Le CNAL ne veut plus réduire la laïcité à la seule question scolaire". Le Monde, Paris, 15 mars, 1988. 
Bousseageon, Alexandre. "L'impossible ramadan". Témoignage chrétien, Paris, 20 aout, 1979.

Chetucci Claude, Hatzfeld Nicolas. "L'administration du Travail et les conflits collectifs: Citroën et Talbot (1982-1983)". Travail et Emploi No. 111 (2007): 31-38.

Collectif. "Quand la laïcité se voile la face...". Rouge et vert, Paris, 18 novembre, 1989.

Durand-Souffland, Jean-Marie. "Musulmans en France". Le Monde, Paris, 13 juillet, 1983.

Flandre Emmanuel, Pattacini Jean-Claude. "Paris: l'islam des pauvres". L'express, Paris, 15 juillet, 1983.

Goguel Anne-Marie. “Un jugement de Salomon”. Réforme, Paris, 4 août, 1984.

Grosser Alfred. “L'avenir de la liberté”. Le Monde, Paris, 11 janvier, 1977. Lorcerie Françoise. "La 'loi sur le voile': une entreprise politique”. Droit et société No. 68 (2008), 53-74.

Poulat Emile. "Liberté scolaire, laïcité républicaine”. La Pensée No. 237 (1984): 42-52.

Rayman Béatrice, Rochal Albert. "Intégration ou assimilation?”. Critique communiste No. 91 (1989).

Tersigni Simona. "Prendre le foulard": les logiques antagoniques de la revendication". Mouvements No. 30 (2003): 116-122.

Pierre Tournemire, "Une loi qui serait inopportune", Idées en mouvement No. 110, (2003).

"Note de synthèse du Service d'information et de diffusion", 6 novembre, 1989 (104 APO 9, Office universitaire de recherche socialiste).

\section{Fuentes de Archivo}

Fonds du cabinet du Ministère de l'Education nationale, 1984-1986 (série SV), Paris: Fondation nationale des sciences politiques.

Fonds du cabinet du Ministère de l'Education nationale, 1984-1986 (série 19880108), Peyrefitte: Archives nationales.

Fonds Luc Soubré, 1989-1992 (série 104 APO), Paris: Office universitaire de recherche socialiste.

Para citar este artículo: Ferhat, Ismail "De l'enseignement catholique au foulard musulman: la laïcité scolaire entre débats et mutations en France, 1984-2004", Historia Caribe Vol. XII No. 30 (Enero-Junio 2017): 245266. DOI: http://dx.doi.org/10.15648/hc.30.2016.9 